\title{
Diagnosis of diffuse parenchymal lung diseases using transbronchial cryobiopsy guided by endobronchial ultrasound compared to clinicoradiological diagnosis
}

\author{
Xiao $\mathrm{Li}^{1}$, Jinbing $\mathrm{Pan}^{1}$, Yun $\mathrm{Ma}^{1}$, Ying Ren ${ }^{2}$, Bu-Lang Gao ${ }^{1} \wedge$ \\ ${ }^{1}$ Department of Respiratory and Critical Care Medicine, Henan Provincial People's Hospital, Zhengzhou, China; ${ }^{2}$ Department of Pathology, Henan \\ Provincial People's Hospital, Zhengzhou, China
}

Contributions: (I) Conception and design: J Pan, X Li; (II) Administrative support: Y Ma; (III) Provision of study materials or patients: X Li, J Pan, Y Ma; (IV) Collection and assembly of data: J Pan, X Li, BL Gao; (V) Data analysis and interpretation: X Li, J Pan, Y Ma, Y Ren, BL Gao. (VI) Manuscript writing: All authors. (VII) Final approval of manuscript: All authors.

Correspondence to: Jinbing Pan. Department of Respiratory and Critical Care Medicine, Henan Provincial People's Hospital, Henan University, 7 Weiwu Road, Zhengzhou, China. Email: jinwu9636@126.com.

Background: This study aimed to evaluate the safety and effect of transbronchial cryobiopsy guided by radial probe endobronchial ultrasound (RP-EBUS) compared with clinicoradiological diagnoses in diffuse parenchymal lung diseases (DPLDs).

Methods: A total of 60 patients with DPLDs confirmed by chest computed tomography (CT) who underwent transbronchial lung cryobiopsy guided by RP-EBUS were enrolled. The ultrasound images were obtained and identified together with corresponding chest CT characteristics. The cryobiopsy samples were evaluated histopathologically and compared with CT imaging, and the complications were analyzed.

Results: The multidisciplinary diagnosis was clear in 51 (85\%) participants but unclear in the remaining $9(15 \%)$ participants. In transbronchial cryobiopsy guided by RP-EBUS, 36 (60\%) participants had the biopsy in 1 lobe while 24 (40\%) had a biopsy in 2 different lobes, with a mean biopsy specimen size of $43.17 \pm 15.25 \mathrm{~mm}^{2}$. The histopathologic diagnosis based on biopsy confirmed the preprocedural clinicoradiological diagnosis in $51(85 \%)$ patients and clarified the diagnosis in the other 9 patients with unclear clinicoradiological diagnosis, including alveolated lung parenchyma with interstitial chronic inflammation in $4(6.7 \%)$ cases and chronic bronchiolitis and interstitial lymphocytic infiltrates in the other 5 (8.3\%). Intraprocedural complications occurred in 57 (95\%) patients, including pneumothorax in $9(15 \%)$, bleeding in 47 (78.3\%), and hypoxemia in 1 (1.7\%). The ultrasound images of DPLDs were normal, mesh $(\mathrm{n}=24)$, nodular $(\mathrm{n}=9)$, and alveolar type $(\mathrm{n}=27)$.

Conclusions: Transbronchial cryobiopsy guided by RP-EBUS is safe and effective and can supply additional information to the clinicoradiological approach for correct diagnosis of DPLDs.

Keywords: Transbronchial cryobiopsy; diffuse lung diseases; diffuse parenchymal lung diseases (DPLDs); radial probe endobronchial ultrasound (RP-EBUS); computed tomography (CT)

Submitted Mar 08, 2021. Accepted for publication Sep 03, 2021.

doi: 10.21037/qims-21-255

View this article at: https://dx.doi.org/10.21037/qims-21-255

\footnotetext{
^ ORCID: 0000-0003-4668-0737.
} 


\section{Introduction}

Diffuse parenchymal lung diseases (DPLDs) are a group of diverse pulmonary disorders with similar clinical, radiologic, and lung function presentations. They can be caused by a variety of diseases, involving a complex process for clinical diagnosis. Histological analysis of the lung parenchyma is needed to reach a conclusive and specific diagnosis of DPLD (1). Surgical lung biopsies have been considered the gold standard for correct diagnosis. Approximately $22-54 \%$ of patients with idiopathic pulmonary fibrosis (IPF) undergo surgical lung biopsy for diagnosis when classic radiology cannot determine the nature of disease $(2,3)$. However, surgical lung biopsy, which requires endotracheal intubation, chest tube placement, and hospitalization, has the risk of air leakage, bronchopleural fistula, and even death $(4,5)$. This provides a strong rationale for the use of less invasive techniques of lung biopsy for correct diagnosis.

The diagnostic yield of endobronchial procedures has been shown to be low, mostly due to the small size of biopsy specimens obtained with biopsy forceps through bronchoscopy. In a study by Shim et al., only 3 biopsy specimens obtained by transbronchial lung biopsy met the usual interstitial pneumonia (UIP) diagnostic criteria in 32 patients with this disease, confirmed by surgical lung biopsy (6). Another study reported that among 801 DPLD patients, only 232 cases $(29 \%)$ had a specific histopathological diagnosis made via biopsy forceps, with most of the diagnoses being malignancy (7). Compared with the endobronchial biopsy forceps, transbronchial cryobiopsy is a feasible technique for obtaining larger and better samples than those of endobronchial biopsy. One study found surgical lung biopsy to be unnecessary in $75 \%$ cases of interstitial lung disease (ILD) compared with transbronchial cryobiopsy (8). Transbronchial cryobiopsy can significantly improve the clinical diagnostic yield but may be associated with a higher incidence of complications, especially the risk of massive hemorrhage (9-11). Ultrasound can be used to identify peribronchial parenchymal tissues and minimize risk of hemorrhage during peripheral lung cryobiopsy in patients with suspected ILD (12). The use of radial probe endobronchial ultrasound (RP-EBUS) is essential for selecting the correct location of pulmonary lesions for accurate diagnosis in bronchoscopy. Currently, however, there have been few reports addressing the imaging features of DPLDs on RP-EBUS and the feasibility and diagnostic yield of RP-EBUS-guided bronchoscopic cryobiopsy. It was hypothesized that RP-EBUS could be used to investigate the imaging features of DPLDs and that bronchoscopic cryobiopsy with the guidance of RP-EBUS could be safely performed to correctly diagnose DPLDs. This study was consequently conducted to examine the diagnostic yield of bronchoscopic cryobiopsy guided by RP-EBUS in patients with DPLDs and reveal the ultrasonic imaging features of DPLDs on EP-EBUS.

\section{Methods}

\section{Study population}

This study was conducted in accordance with the Declaration of Helsinki (as revised in 2013). The study was approved by institutional ethics committee of Henan Provincial People's Hospital (2011332083) and informed consent was provided by all participants. All methods were performed in accordance with relevant guidelines and regulations $(13,14)$. From May 2016 to January 2019, patients with DPLDs referred to our hospital for bronchoscopy were retrospectively enrolled. Clinical information was analyzed, including medical history, routine blood test (routine lab test, blood coagulation index, autoantibodies, and allergens), high resolution chest computed tomography (HRCT), pulmonary function, and arterial blood gas analysis. The inclusion criteria were patients with DPLDs who had undergone bronchoscopic cryobiopsy guided by RP-EBUS with complete clinical data. The exclusion criteria were patients with anticoagulation therapy, presence of a coagulation disorder (platelets $<50,000$ cells $/ \mathrm{mm}^{3}$, international normalized ratio $>1.5$, and activated partial thromboplastin time $>50 \mathrm{~s}$ ), hypoxemia $\left(\mathrm{PO}_{2}<60 \mathrm{mmHg}\right.$ ), severe respiratory impairment (forced expiratory volume in $1 \mathrm{~s}<50 \%$, total lung capacity $<50 \%$, diffusing capacity of carbon monoxide $<50 \%$ of reference), and chronic cardiovascular disease.

\section{Instruments}

The instruments used included BF-1T260 and BF1T60 flexible bronchoscopes (Olympus, Tokyo, Japan), an endoscopic ultrasound system (EU-M2000; Olympus, Japan) equipped with a $20-\mathrm{MHz}$ miniature radial probe (UM-S20-20R; Olympus, Japan), a cryotherapy instrument, and a flexible cryoprobe with a diameter of 1.8 or $1.5 \mathrm{~mm}$ and a length of 1,200 mm (Kooland, Beijing, China). The guide sheath measured $2.55 \mathrm{~mm}$ (K-203 GS Kit; Olympus, Japan) or $1.95 \mathrm{~mm}$ in diameter (K-201 GS Kit; Olympus, 
Japan).

\section{Anesthetic administration}

The procedure was performed under general anesthesia, followed by insertion of an appropriate laryngeal mask which was connected to the anesthesia machine with a $\mathrm{T}$ connector extension tube. Propofol $(0.5-1 \mathrm{mg} / \mathrm{kg})$ was added according to the operation schedule, and $2 \%$ lidocaine was injected into the tracheobronchial tree (less than $40 \mathrm{~mL}$ in total) in the process. After the cryobiopsy, the patient was intravenously administrated with $0.1 \mathrm{mg}$ naloxone, $0.5 \mathrm{mg}$ atropine, and $1 \mathrm{mg}$ neostigmine. The laryngeal mask was removed when the muscle strength and consciousness were restored, and the patients were sent into the resuscitation ward for further observation and monitoring of electrocardiogram, blood oxygen saturations, and blood pressure throughout the whole process.

\section{Non-real-time EBUS-guided cryobiopsy}

A flexible bronchoscope was inserted through a $\mathrm{T}$ connector extension tube into the subsegmental branches of the target lobe according to CT imaging. After routine bronchoscopy check, the RP-EBUS was inserted into the segment to determine the location of lesion based on the ultrasound characteristics through the guide sheath. Then, the probe was removed while keeping the guide sheath in place for the cryobiopsy. An X-ray examination was performed to determine the position of the cryoprobe for insertion, with an optimal distance of approximately $10 \mathrm{~mm}$ from the cryoprobe to the thoracic wall. After cryobiopsy, the cryoprobe was cooled with carbon dioxide as the refrigerant for approximately $3-5 \mathrm{~s}$ before withdrawal of the cryoprobe, guide sheath, and the bronchoscope. The frozen specimen was thawed in saline before being fixed with formalin. Another bronchoscope was inserted immediately to observe possible hemorrhage during the thawing period, and further treatment was performed if there was hemorrhage in the biopsy location. Hemostatic drugs and a Fogarty balloon were prepared before operation. The number of biopsy samples was determined based on the lesion size. Chest $\mathrm{X}$-ray was used to exclude pneumothorax within $2 \mathrm{~h}$ after the operation.

\section{Processing the histological samples}

Specimens were fixed in $4 \%$ formalin and embedded in paraffin. Each specimen was evaluated by two pathologists. The diameter, area, and number of specimens and artificial artifacts (atelectasis and broken tissue block) were recorded. Clinical and radiological features were provided to the pathologist for reference. Multidisciplinary diagnosis was made according to clinical, radiographic, and pathologic findings.

\section{Treatment complications}

After cryobiopsy, patients were usually observed for 4 hours before their return to the general ward. Bleeding was classified on an adapted scale: grade 0 , no bleeding; grade 1 , bleeding requiring suction to clear but no endoscopic treatment; grade 2, bleeding requiring endoscopic treatment (bronchial occlusion-collapse and/or instillation of ice-cold saline); grade 3, severe bleeding which may cause hemodynamic or respiratory instability that could not be controlled by endoscopic procedures and requiring surgical interventions or admission to the intensive care unit (ICU) (15). If the patients developed chest pain or distress, they were immediately examined by auscultation to exclude pneumothorax or hemothorax, and X-ray examination was performed when necessary. If the lung was compressed more than $30 \%$, a drain was placed.

\section{Statistical analysis}

The SPSS 13 software (IBM Corp., Armonk, NY, USA) was used for statistical analysis. Measurement data are expressed as mean \pm standard deviation and were analyzed with the Student's $t$-test if normally distributed. If the distribution was skewed, measurement data were tested with the chi-squared test and are presented as median \pm range. Enumeration data were tested with the chi-squared test and are presented as numbers and percentages. Significance of the $\mathrm{P}$ value was set at $<0.05$.

\section{Results}

A total of 60 patients who had undergone transbronchial cryobiopsy guided by RP-EBUS were enrolled, including 29 males and 31 females, with an age range of 27 to 72 years (mean 51.08 12.49 ; Table 1 ). The preprocedural clinicoradiological diagnosis was clear in $51(85 \%)$ patients but unclear in the other $9(15 \%$; Tables 1,2$)$, an indicated IPF in $19(31.7 \%)$ cases, nonspecific interstitial pneumonia in $13(21.7 \%)$, adenocarcinoma in $5(8.3 \%)$, organizing 
pneumonia in $3(5 \%)$, desquamative interstitial pneumonitis in $2(3.3 \%)$, sarcoidosis in $2(3.3 \%)$, drug interstitial pneumonia in $1(1.7 \%)$, tuberculosis in $2(3.3 \%)$, diffuse panbronchiolitis in $2(3.3 \%)$, and pulmonary infection in 2

Table 1 Clinical data

\begin{tabular}{lc}
\hline Variables & Data \\
\hline Participants & \\
Age (year) & $29.08 \pm 12.49[27-72]$ \\
Male/female & \\
Biopsy & $36(60 \%)$ \\
One lobe & $24(40 \%)$ \\
Two different lobes & $43.17 \pm 15.25$ \\
Size of specimen (mm $\left.{ }^{2}\right)$ & \\
Diagnosis & $51(85 \%)$ \\
Multidisciplinary diagnosis & $9(15 \%)$ \\
Nondiagnostic & \\
Complications & $9(15 \%)$ \\
Pneumothorax & $13(21.7 \%)$ \\
Bleeding grade 0 & $42(70 \%)$ \\
Bleeding grade 1 & $5(8.3 \%)$ \\
Bleeding grade 2 & $1(1.6)$ \\
Hypoxemia & \\
\hline
\end{tabular}

(3.3\%). In transbronchial cryobiopsy guided by RP-EBUS (Figures 1-3), 36 (60\%) participants had the biopsy in 1 lobe while $24(40 \%)$ had it in 2 different lobes, and the mean size of biopsy specimen was $43.17 \pm 15.25 \mathrm{~mm}^{2}$.

The histopathologic diagnosis based on the biopsy tissue confirmed the clinicoradiological diagnosis in 51 (85\%) patients and clarified the diagnosis in the other 9 patients with unclear clinicoradiological diagnosis. Unclear preprocedural diagnoses were shown on transbronchial cryobiopsy to have alveolate lung parenchyma with interstitial chronic inflammation in 4 (6.7\%) cases and chronic bronchiolitis and interstitial lymphocytic infiltrates in the other $5(8.3 \%)$; however, some of these cryobiopsy findings might not have been specific and required further confirmation.

During the transbronchial cryobiopsy procedure, 57 complication events occurred in 47 (78.3\%) patients, including pneumothorax in 9 (15\%), bleeding in 47 $(78.3 \%)$, and hypoxemia in 1 (1.7\%) (Table 1$)$. Bleeding was the most common complication, including grade 1 bleeding in $41(68.3 \%)$ cases and grade 2 in 5 (8.3\%); only $13(21.7 \%)$ patients experience no bleeding during the procedure. However, no severe bleeding occurred, and thus no interventional therapy or surgery was required. Apart from these nonsevere bleeding complications, other complications (pneumothorax and hypoxemia) occurred, accounted for $16.7 \%$ of complications. Pneumothorax in 9 cases was cured after drainage. One patient was transferred to the ICU for 12 hours due to hypoxemia.

Table 2 Clinicoradiological and histopathologic diagnosis based on biopsy

\begin{tabular}{ll}
\hline Clinicoradiological diagnosis $(n=60)$ & Histopathologic diagnosis $($ biopsy, $n=60)$ \\
\hline Idiopathic pulmonary fibrosis $(n=19,31.7 \%)$ & Usual interstitial pneumonia $(n=19,31.7 \%)$ \\
Nonspecific interstitial pneumonia $(n=13,21.7 \%)$ & Nonspecific interstitial pneumonia $(n=13,21.7 \%)$ \\
Adenocarcinoma $(n=5,8.3 \%)$ & Adenocarcinoma $(n=5,8.3 \%)$ \\
Organizing pneumonia $(n=3,5 \%)$ & Organizing pneumonia $(n=3,5 \%)$ \\
Desquamative interstitial pneumonitis $(n=2,3.3 \%)$ & Desquamative interstitial pneumonitis $(n=2,3.3 \%)$ \\
Sarcoidosis $(n=2,3.3 \%)$ and drug interstitial pneumonia & Nonnecrotizing granulomatous inflammation $(n=3,5 \%)$ \\
(n=1, $1.7 \%)$ & \\
Tuberculosis $(n=2,3.3 \%)$ & Necrotizing granulomatous inflammation $(n=2,3.3 \%)$ \\
Diffuse panbronchiolitis $(n=2,3.3 \%)$ & Respiratory bronchiolitis $(n=2,3.3 \%)$ \\
Pulmonary infection $(n=2,3.3 \%)$ & Acute fibrinous and bronchiolitis $(n=2,3.3 \%)$ \\
Unclear $(n=4,6.7 \%)$ & Alveolate lung parenchyma with interstitial chronic inflammation $(n=4,6.7 \%)$ \\
Unclear $(n=5,8.3)$ & Chronic bronchiolitis and interstitial lymphocytic infiltrates $(n=5,8.3 \%)$ \\
\hline
\end{tabular}



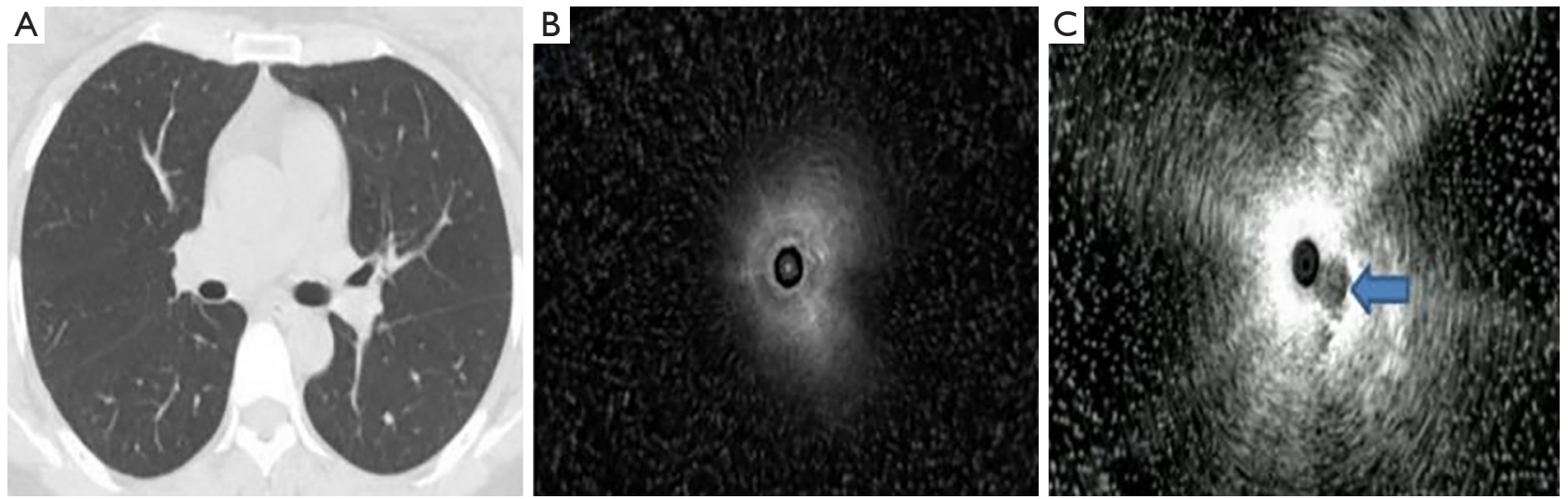

Figure 1 The EBUS images of a normal lung. (A) A chest CT image of normal lung tissue; (B) a diffuse fine granular pattern on EBUS, with a hyperechoic area in the center and gradually decreased density in the peripheral area; (C) angiography findings on ultrasound showing density, a clear boundary, and hypoechoic vessels (arrow). EBUS, endobronchial ultrasound.
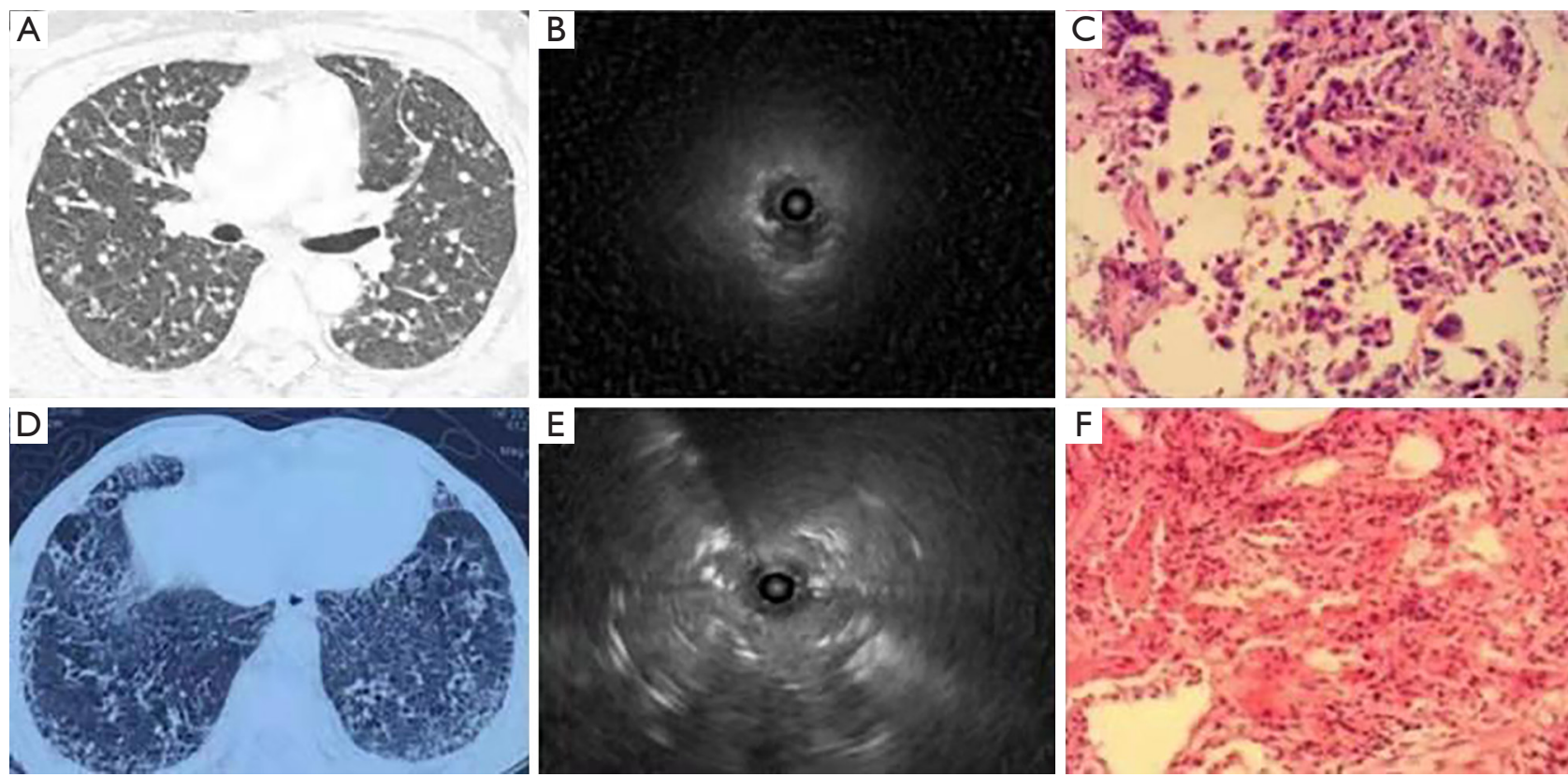

Figure 2 The EBUS images of nodular lesions and reticulation lesions on chest CT images. (A) Multiple solid and well-defined nodules in both lungs on chest CT imaging; (B) when the echo probe was adjacent to the lesion, low-level echo areas were surrounded by a clearly hypoechoic border on ultrasound; (C) adenocarcinoma cells in the lungs (HE, $\times 100)$; (D) reticulation at both lower lungs and local honeycomb changes on chest CT imaging; (E) scattered points and linear hyperechoic regions in a field of whitish acoustic shadows on EBUS; (F) pulmonary fibrosis $(\mathrm{HE}, \times 100$ ). EBUS, endobronchial ultrasound; CT, computed tomography; HE, hematoxylin and eosin staining.

For the 9 patients with unclear preprocedural diagnoses, follow-up was performed for 6-12 months: 3 patients died, 2 were lost to follow-up, 2 were confirmed to have pulmonary infection which was responsive to antibacterial treatment,
1 was confirmed to have allergic pneumonia, and 1 was confirmed with organizing pneumonia. These 2 participants with pneumonia were responsive to glucocorticoid therapy. Both participants were given methylprednisolone orally, 

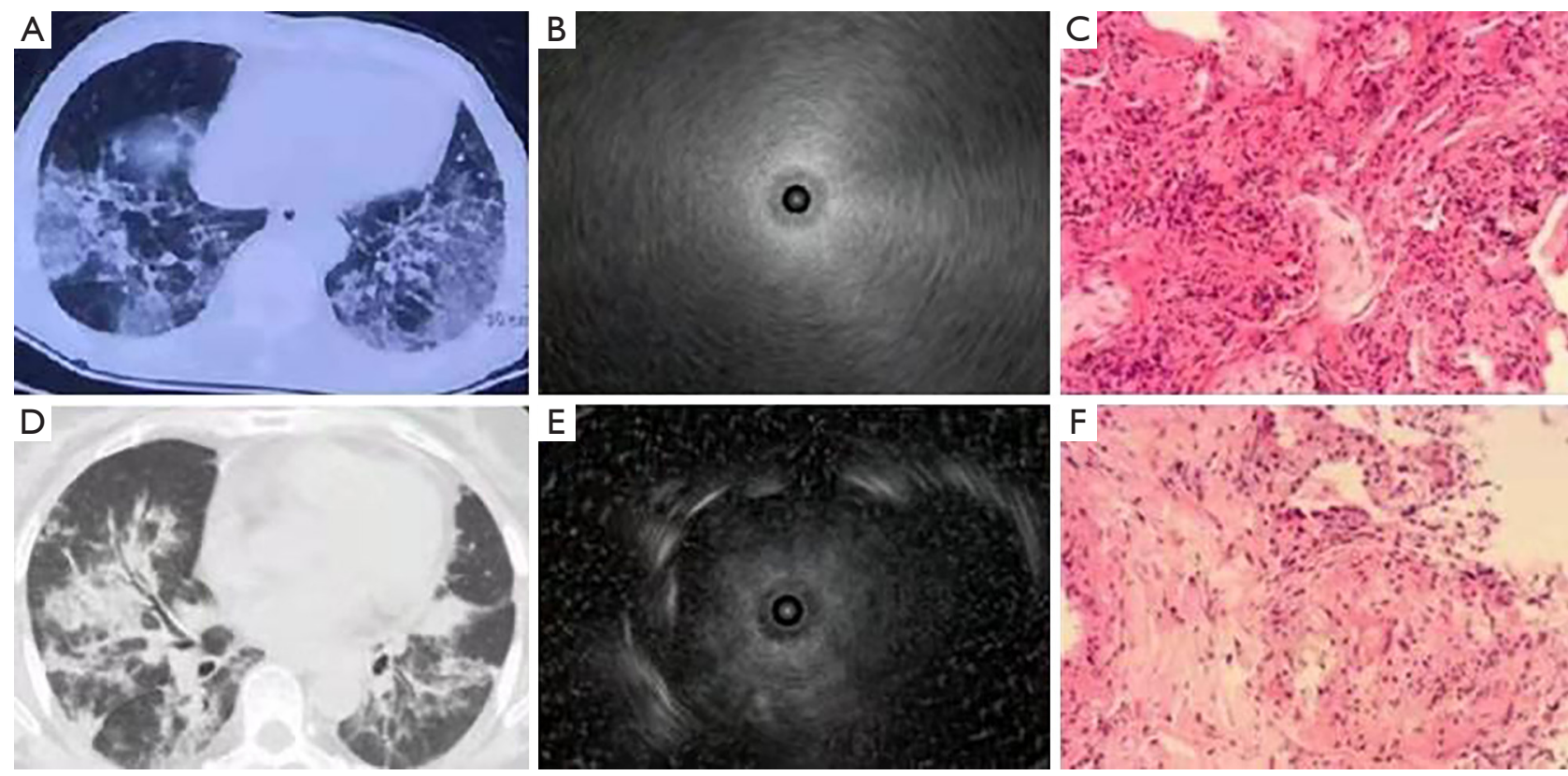

Figure 3 The EBUS images of alveolar lesions on chest CT imaging. (A) Ground-glass lesions with bilateral distribution on chest CT imaging; (B) a blizzard sign; (C) organizing pneumonia in pathology (HE, $\times 100)$; (D) consolidation at the middle lobe of the right lung with an air bronchogram on chest CT imaging; (E) low-echo areas with or without a clear boundary within the solid shadow on ultrasound using an ultrasound probe; (F) granulomatous inflammation was considered to sarcoidosis in pathology (HE, $\times 100)$. EBUS, endobronchial ultrasound; CT, computed tomography; HE, hematoxylin and eosin staining.

with an initial dose in the patient with allergic pneumonia of $48 \mathrm{mg}$ once a day for 2 weeks, which was decreased to $16 \mathrm{mg}$ once a day for the next 2 weeks, and decreased thereon by $2 \mathrm{mg}$ every week until withdrawal. The dose of methylprednisolone in the patient with organizing pneumonia was initially $52 \mathrm{mg}$ once a day for 4 weeks, then decreased to $12 \mathrm{mg}$ once a day for 6 months, and finally reduced to $2 \mathrm{mg}$ once a week until withdrawal.

During the ultrasound examination (Figures 1-3), blood vessels in the lesion were detected (Figure 1C). According to the imaging presentations on chest CT, DPLDs were classified into 4 types: normal (Figure 1A), nodular (Figure $2 A$ ), mesh (Figure 2D), and alveolar (Figure $3 A, 3 D$ ). On ultrasound imaging, the normal type was characterized by a diffuse fine granular pattern, with a hyperechoic area in the center but gradually decreased density in the peripheral area (Figure 1B). The nodular type in 9 patients showed a low-level echo area with or without hyperechoic dots or lines surrounded by a clear high echo border (Figure 2B, with the ultrasound probe adjacent to the lesion). The mesh type in 24 patients was of diffuse dense points and lines of hyperechogenicity scattered in a field of whitish acoustic shadow (Figure 2E). The alveolar type in 27 patients was composed of three forms: (I) a mild increase in the intensity and radius of the whitish acoustic shadow (blizzard sign) (16) when the lesion was of pure ground-glass change (Figure $3 B$ ), (II) some hyperechoic dots and linear arcs distributed irregularly within a blizzard for part-solid ground-glass opacity (GGO), and (III) a low echo area with or without a clear boundary when the pulmonary consolidation or mass lesion was of homogeneous density on CT imaging. When the lesion was heterogeneous, scattered points or linear hyperechoic areas were seen in the context of low-level echo areas (Figure $3 E$, with the echo probe located within the solid shadow). The third image form had the combined characteristics of the former 2 when patchy shadows were present on chest CT imaging.

\section{Discussion}

In this study, the safety and effect of transbronchial RPEBUS-guided cryobiopsy were investigated in comparison with CT imaging in diagnosing DPLDs, and it was found that transbronchial RP-EBUS-guided cryobiopsy was safe 
and effective addition to the correct diagnosis of DPLDs by providing additional information to the clinicoradiological approach to diagnosis.

On CT imaging, DPLDs may be nodular, mesh, and alveolar in bilateral lungs (17). However, these patterns of pulmonary imaging may represent different diseases, such as pulmonary carcinoma, tuberculosis, or groundglass nodules of glucose metabolism $(18,19)$. Thus, the clinicoradiological approach based on imaging features and clinical data could not reach a correct diagnosis in 9 $(15 \%)$ patients although most patients $(\mathrm{n}=51,85 \%)$ were correctly diagnosed in our study. Transbronchial lung biopsy may obtain small specimens or miss the actual tissue of pulmonary lesions, preventing the ability to attain a correct diagnosis in most patients $(6,7)$. In one study, surgical lung biopsy was deemed unnecessary in $75 \%$ cases of ILD compared with transbronchial cryobiopsy (8). The pathological diagnosis rate of interstitial pneumonia by cryobiopsy reached $74.4 \%$ in a study with the final diagnosis rate by cryobiopsy of $51.3 \%$, which was higher than that of conventional forceps biopsy (20). In other research, the area of the cryobiopsy group was reported to be $14.7 \pm 11 \mathrm{~mm}^{2}$, greater than that of the forceps biopsy $\left(3.3 \pm 4.1 \mathrm{~mm}^{2}\right)$, and there was no significant difference in complications between the cryobiopsy and conventional forcep biopsy groups. In a systematic review and meta-analysis, Dhooria et al. reviewed 14 studies on cryobiopsy involving 1,183 patients with DPLD, and the results were similar to those of previous research (20). In this study (21), the pooled diagnostic yield of cryo-transbronchial lung biopsy was $76.9 \%$ [95\% confidence interval (CI): 67.2-85.3] when only definitive diagnoses were taken into consideration and $85.9 \%$ (95\% CI: 78.2-92.2) when both definitive and probable diagnoses were considered, and the diagnostic yield of cryotransbronchial lung biopsy $(86.3 ; 95 \%$ CI: 80.2-90.8) was significantly greater than that of flexible forceps biopsy (56.5\%, 95\% CI: $27.5-83.2)$ with an odds ratio of 6.7 (95\% CI: 3.6-12.4). The meta-analysis (21) also demonstrated that the size of samples obtained with cryo-transbronchial lung biopsy was significantly greater than that obtained with flexible forceps biopsy (20.4vs. $\left.4.3 \mathrm{~mm}^{2}, \mathrm{P}=0.005\right)$. After analyzing 8 studies of cryobiopsy for DPLDs or cancer patients, Ganganah et al. (22) found that cryoproberetrieved specimens were larger and more effective than forceps biopsy specimens in obtaining sufficient tissues for histologic diagnosis, even though the amount and rate of bleeding were higher than those of conventional forceps biopsy. One study confirmed the safety and effectiveness of transbronchial RP-EBUS-guided cryobiopsy and revealed that it had clarified some ill-defined diagnoses. No severe hemorrhage was present in our study; however, in order to prevent potential massive hemorrhage, it is necessary to detect and avoid blood vessels in the area of cryobiopsy.

The RP-EBUS has some advantages in the guidance of cryobiopsy as it has a sensor of $20 \mathrm{MHz}$ frequency, with the minimum resolvable range of $\sim 77 \mu \mathrm{m}$, which is smaller than the normal alveolar diameter $(\sim 200 \mu \mathrm{m})$. The echoic images of diseases may represent mixed lesions and reflect the pulmonary structures, and different echoic patterns of DPLDs may be related to the degree of structural changes caused by lung diseases (23). Peripheral pulmonary lesions biopsy can be located and biopsied by RP-EBUS through a sheath tube (24), and when peripheral pulmonary lesions are cryobiopsied under ultrasound guidance, the accuracy of biopsy and diagnosis rate can be increased and the bleeding rate decreased because blood vessels can be detected and avoided $(24,25)$. The RP-EBUS can assist in the selection of a less vascular area for cryobiopsy, thus resulting in a decreased risk of hemorrhage. It has been reported that radial ultrasound could be used to identify at least $1 \mathrm{~cm}$ of peribronchial lung parenchyma with low vascularity to minimize risk of hemorrhage during peripheral lung cryobiopsy in patients with suspected ILD (12). The abovementioned studies suggest that ultrasonography can be used to reduce the risk of bleeding; however, no studies have described the image features of RP-EBUS in DPLDs.

Aside from the normal type, the types of DPLDs on CT imaging were nodular, mesh, and alveolar, and the ultrasound patterns of DPLDs were also classified into the corresponding types, with the exact image presentations depending on the size and density of lesion as well as the relationship between the lesion and the ultrasound probe. In the normal ultrasound type, ultrasound could not show the alveolar epithelia because of gas interference. In the nodular type, when the ultrasound probe was positioned adjacent to or outside the lesion, a low-echoic area was shown with a clear boundary under the background of the normal lung. The surrounded boundary was a strong-reflected interface produced between the aerated lung and the lesion. In this type of lesion, the biopsy specimens may not be obtained with conventional forceps (26), but can be extracted by cryo-adhesion when transbronchial cryobiopsy is performed because the tissue surrounding the ultrasound probe tip is frozen. This might be one of the main advantages of the transbronchial cryobiopsy, especially in lesions where the EBUS cannot be performed within the lesion (11). 
In the mesh type lesion, diffuse dense points and linear hyperechoic regions were scattered in the granular context, which is probably caused by extensive reflected interfaces produced between the heterogeneous pulmonary interstitial tissues, with the alveolar structure being largely damaged. The alveolar type had 3 different manifestations depending on different pathological periods. In the beginning of alveolar edema, ground-glass change was present on CT images, resulting in the ultrasound image pattern of blizzard signs described by Izumo (16). When alveolar edema became aggravated, pulmonary consolidation occurred, and low-echo areas with or without continuous hyperechoic margins were evident on ultrasound when the ultrasonic probe was centered on the lesions. In the alveolar type, internal echoes would be uniformly dense when the region was homogeneous on CT imaging, and some hyperechoic dots and linear areas appeared scattered in the background of low echo when the lesion was inhomogeneous.

Mixed blizzard imaging is caused by the heterogenicity of the lesions which contain gas and blood vessels, and the characteristic of mixed blizzard ultrasound is inhomogeneous echo, with hyperechoic dots inside the lesion. The hyperecho in the ultrasound image in Figure $2 E$ may have been caused by thickening of lung stroma mixed with air, with the ultrasound image being characterized by points and linear hyperechoic features. Our study did not continue to use the traditional ultrasound appellation, but described the image features for easy understanding. The research object of this study was the DPLDs which may have complex and diverse ultrasound images, quite different from those of lesions with solid components or gascontaining peripheral lung cancers. Moreover, DPLDs may be very small, and the ultrasound probe can only reach the tissues adjacent to these small lesions, which is the reason why the ultrasound image may reflect the area adjacent to the lesion or within the lesion.

Early studies performed cryobiopsy through rigid bronchoscopes or endotracheal tubes because a bronchoscope could be navigated into the lung more easily, avoiding injury to vocal cords and possible bleeding $(11,27)$; however, a deeper level of anesthesia was needed. In our study of cryobiopsy, the bronchoscopy was performed under the laryngeal mask airway, which did not require deeper anesthesia, leading to fast patient recovery and minimal risk. We did not observe any injury to the vocal cords or larynx when tissues in the airways were frozen and withdrawn together with the bronchoscope under laryngeal mask. We biopsied 2 lobes if the imaging showed different lesions in different lobes of the lung, such as ground glass in the right upper lung and consolidation in the right lower lung. If the biopsied material from one lobe was not ideal, sampling in another lobe was necessary.

Some limitations existed in our study, including its use of a retrospective design, a single center, and a small cohort of exclusively Chinese patients, along with a lack of nonrandomization and a control group for comparison with other non-diffuse diseases, may restrict the interpretation and generalization of our conclusions. Future prospective, multi-center, multi-race, randomized, and controlled studies with a large cohort of participants are needed to resolve these issues to produce better outcomes.

\section{Conclusions}

Cryobiopsy under the guidance of RP-EBUS can be safely used to correctly diagnose diffuse lung diseases by providing additional information to the clinicoradiological approach of diagnosis and avoiding blood vessels and possible massive hemorrhage.

\section{Acknowledgments}

Funding: None.

\section{Footnote}

Conflicts of Interest: All authors have completed the ICMJE uniform disclosure form (available at https://dx.doi. org/10.21037/qims-21-255). The authors have no conflicts of interest to declare.

Ethical Statement: The authors are accountable for all aspects of the work in ensuring that questions related to the accuracy or integrity of any part of the work are appropriately investigated and resolved. The study was conducted in accordance with the Declaration of Helsinki (as revised in 2013). The study was approved by institutional ethics committee of Henan Provincial People's Hospital (No. 2011332083), and informed consent was provided by all participants.

Open Access Statement: This is an Open Access article distributed in accordance with the Creative Commons Attribution-NonCommercial-NoDerivs 4.0 International License (CC BY-NC-ND 4.0), which permits the noncommercial replication and distribution of the article with 
the strict proviso that no changes or edits are made and the original work is properly cited (including links to both the formal publication through the relevant DOI and the license). See: https://creativecommons.org/licenses/by-nc-nd/4.0/.

\section{References}

1. European Respiratory Society. American Thoracic Society/European Respiratory Society International Multidisciplinary Consensus Classification of the Idiopathic Interstitial Pneumonias. This joint statement of the American Thoracic Society (ATS), and the European Respiratory Society (ERS) was adopted by the ATS board of directors, June 2001 and by the ERS Executive Committee, June 2001. Am J Respir Crit Care Med 2002;165:277-304.

2. Noble PW, Albera C, Bradford WZ, Costabel U, Glassberg MK, Kardatzke D, King TE Jr, Lancaster L, Sahn SA, Szwarcberg J, Valeyre D, du Bois RM; CAPACITY Study Group. Pirfenidone in patients with idiopathic pulmonary fibrosis (CAPACITY): two randomised trials. Lancet 2011;377:1760-9.

3. Richeldi L, Costabel U, Selman M, Kim DS, Hansell DM, Nicholson AG, Brown KK, Flaherty KR, Noble PW, Raghu G, Brun M, Gupta A, Juhel N, Klüglich M, du Bois RM. Efficacy of a tyrosine kinase inhibitor in idiopathic pulmonary fibrosis. N Engl J Med 2011;365:1079-87.

4. Ayed AK, Raghunathan R. Thoracoscopy versus open lung biopsy in the diagnosis of interstitial lung disease: a randomised controlled trial. J R Coll Surg Edinb 2000;45:159-63.

5. Utz JP, Ryu JH, Douglas WW, Hartman TE, Tazelaar HD, Myers JL, Allen MS, Schroeder DR. High shortterm mortality following lung biopsy for usual interstitial pneumonia. Eur Respir J 2001;17:175-9.

6. Shim HS, Park MS, Park IK. Histopathologic findings of transbronchial biopsy in usual interstitial pneumonia. Pathol Int 2010;60:373-7.

7. Poletti V, Patelli M, Poggi S, Bertanti T, Spiga L, Ferracini R. Transbronchial lung biopsy and bronchoalveolar lavage in diagnosis of diffuse infiltrative lung diseases. Respiration 1988;54 Suppl 1:66-72.

8. Hagmeyer L, Theegarten D, Treml M, Priegnitz C, Randerath W. Validation of transbronchial cryobiopsy in interstitial lung disease - interim analysis of a prospective trial and critical review of the literature. Sarcoidosis Vasc Diffuse Lung Dis 2016;33:2-9.

9. Ravaglia C, Bonifazi M, Wells AU, Tomassetti S, Gurioli
C, Piciucchi S, Dubini A, Tantalocco P, Sanna S, Negri

E, Tramacere I, Ventura VA, Cavazza A, Rossi A, Chilosi M, La Vecchia C, Gasparini S, Poletti V. Safety and Diagnostic Yield of Transbronchial Lung Cryobiopsy in Diffuse Parenchymal Lung Diseases: A Comparative Study versus Video-Assisted Thoracoscopic Lung Biopsy and a Systematic Review of the Literature. Respiration 2016;91:215-27.

10. Hetzel J, Eberhardt R, Herth FJ, Petermann C, Reichle G, Freitag L, et al. Cryobiopsy increases the diagnostic yield of endobronchial biopsy: a multicentre trial. Eur Respir J 2012;39:685-90.

11. Poletti V, Casoni GL, Gurioli C, Ryu JH, Tomassetti S. Lung cryobiopsies: a paradigm shift in diagnostic bronchoscopy? Respirology 2014;19:645-54.

12. Berim IG, Saeed AI, Awab A, Highley A, Colanta A, Chaudry F. Radial Probe Ultrasound-Guided Cryobiopsy. J Bronchology Interv Pulmonol 2017;24:170-3.

13. Chinese Society of Respiratory Diseases. Guidelines on diagnostic flexible bronchoscopy (2008). Zhonghua Jie He He Hu Xi Za Zhi 2008;31:14-7.

14. Chinese Society of Respiratory Diseases. Expert consensus on prevention and treatment of massive hemorrhage related to bronchoscopy diagnosis and treatment. Zhonghua Jie He He Hu Xi Za Zhi 2016;39:588-91.

15. Ernst A, Eberhardt R, Wahidi M, Becker HD, Herth FJ. Effect of routine clopidogrel use on bleeding complications after transbronchial biopsy in humans. Chest 2006;129:734-7.

16. Izumo T, Sasada S, Chavez C, Matsumoto Y, Tsuchida T. Radial endobronchial ultrasound images for ground-glass opacity pulmonary lesions. Eur Respir J 2015;45:1661-8.

17. Dalpiaz G, Maffessanti M. Key pattern. In: Dalpiaz G, Maffessanti M. editors. Diffuse lung diseases: Clinical features, pathology, HRCT. Milan, Italy: Springer-Verlag Italia Press, 2006:8-28.

18. Wen Q, Yue Y, Shang J, Lu X, Gao L, Hou Y. The application of dual-layer spectral detector computed tomography in solitary pulmonary nodule identification. Quant Imaging Med Surg 2021;11:521-32.

19. Shao X, Shao X, Niu R, Jiang Z, Xu M, Wang Y. Investigating the association between ground-glass nodules glucose metabolism and the invasive growth pattern of early lung adenocarcinoma. Quant Imaging Med Surg 2021;11:3506-17.

20. Pajares V, Puzo C, Castillo D, Lerma E, Montero MA, Ramos-Barbón D, Amor-Carro O, Gil de Bernabé A, Franquet T, Plaza V, Hetzel J, Sanchis J, Torrego 
A. Diagnostic yield of transbronchial cryobiopsy in interstitial lung disease: a randomized trial. Respirology 2014;19:900-6.

21. Dhooria S, Sehgal IS, Aggarwal AN, Behera D, Agarwal R. Diagnostic Yield and Safety of Cryoprobe Transbronchial Lung Biopsy in Diffuse Parenchymal Lung Diseases: Systematic Review and Meta-Analysis. Respir Care 2016;61:700-12.

22. Ganganah O, Guo SL, Chiniah M, Li YS. Efficacy and safety of cryobiopsy versus forceps biopsy for interstitial lung diseases and lung tumours: A systematic review and meta-analysis. Respirology 2016;21:834-41.

23. Omori S, Takiguchi Y, Hiroshima K, Tanabe N, Tatsumi K, Kimura H, Nagao K, Kuriyama T. Peripheral pulmonary diseases: evaluation with endobronchial US initial experience. Radiology 2002;224:603-8.

24. Li M, Peng A, Zhang G, Song X, Li J, Tan M, Li X, Liu Y, Wang C. Endobronchial ultrasound transbronchial lung biopsy with guide-sheath for the diagnosis of peripheral pulmonary lesions. Zhonghua Jie He He Hu Xi Za Zhi 2014;37:36-40.

25. Casutt A, Prella M, Beigelman-Aubry C, Fitting JW, Nicod L, Koutsokera A, Lovis A. Fluoroscopic-Guided Radial Endobronchial Ultrasound Without Guide Sheath For Peripheral Pulmonary Lesions: A Safe And Efficient Combination. Arch Bronconeumol 2015;51:338-43.

26. Yamada N, Yamazaki K, Kurimoto N, Asahina H, Kikuchi E, Shinagawa N, Oizumi S, Nishimura M. Factors related to diagnostic yield of transbronchial biopsy using endobronchial ultrasonography with a guide sheath in small peripheral pulmonary lesions. Chest 2007;132:603-8.

27. Yarmus L, Akulian J, Gilbert C, Illei P, Shah P, Merlo C, Orens J, Feller-Kopman D. Cryoprobe transbronchial lung biopsy in patients after lung transplantation: a pilot safety study. Chest 2013;143:621-6.
Cite this article as: Li X, Pan J, Ma Y, Ren Y, Gao BL. Diagnosis of diffuse parenchymal lung diseases using transbronchial cryobiopsy guided by endobronchial ultrasound compared to clinicoradiological diagnosis. Quant Imaging Med Surg 2022;12(2):1139-1148. doi: 10.21037/qims-21-255 